\title{
Coplanar capacitive sensor optimization based on orthogonal experimental design method
}

\author{
Ye Dang ${ }^{1, a}$, Jin Xue ${ }^{1, b}$ and Haiye $Z \mathrm{Zh}^{2}$ \\ ${ }^{1}$ Xi'an Electric power college, Xi'an 710032, China \\ ${ }^{2}$ Beijing University of Technology, Beijing 100124, China

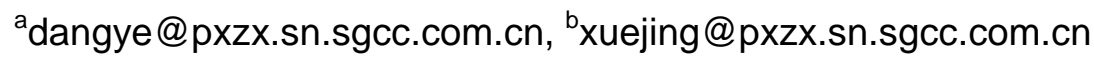

Keywords: coplanar capacitive sensor, fringe capacitance, potential distribution, parameter design.

\begin{abstract}
Coplanar capacitive sensors have been widely applied in many fields with its characterstics of non-invasion, lowcost and fast response. Length and width of sensor electrodes, excitaion frequency are considered to affect the performance of the sensor. In order to improve the sensor performance, and to maximize efficiency of experiments, orthogonal experimental design is introduced. The practical experiments and simulation results are presented at the last. Compared with the full collocation method, the numbers of experiments is reduced by 66\%. The primary and secondary orders of the parameters in this experiment can also determined by this method, and the experimental results are intuitive, reliable, and practical.
\end{abstract}

\section{Introduction}

Capacitive sensing technique has been developed over the past few decades. Parallel-plate sensor is the widely used sort of coplanar sensors which is based on fringe effect existing at the edge of the sensor electrode[1]. The fringe capacitance effect was then used in ECT systems since 1970s[2]. And it has been applied to measurement systems widely with its characteristics of non-invasion, lowcost and fast response. It is one of the most popular sensing techniques in non-destructive testing and evaluation filed.

A technique that uses fringing field interdigital sensors to measure moisture concentration in paper pulp was presented by K.Sundara, and this method can estimate the moisture content in paper pulp at levels as high as 96\% [3]. Pang et al. has presented the principle of non-destructive test of composite materials using the uniplanar capacitive sensor, and the effect of different shield on the detection performance of the sensor was studied but the sensor signal was not researched[4]. Also, the uniplanar capacitance sensor was applied to measure the wood permittivity[5]. A kind of sensor using the fringe electric field was applied to measure the ice layer thickness in the mixture of atmosphere, ice and water [6]. It is easy to know that the fringe capacitance can be used in many systems, but the sensor parameters design, which can affect the sensor performance greatly, also need to be considered.

This paper aims to make tradeoff of capacitive sensor parameters, and determine the effective weight on sensor performance. Working principle of the coplanar capacitive sensor is introduced at the first part. The practical experiments and simulations are proposed based on orthogonal experimental design. The analysis and conclusions of experimental results are presented at the last.

\section{Coplanar Capacitive Sensor Design}

Working principle. A coplanar capacitive sensor consists of two or several electrodes, and an electrostatic field is constructed between the excitation and detective electrodes. One of electrodes for excitation is polarized using an AC or DC voltage, and other electrodes are detectors. The electric charges stored on the surfaces of the plates are different from each other. When a target,such as film, 
comes close or appears on the sensor surface, the electric field line between the excitation and detective electrodes is changed slightly[7], and the fringe capacitance will change, see Fig. 1.

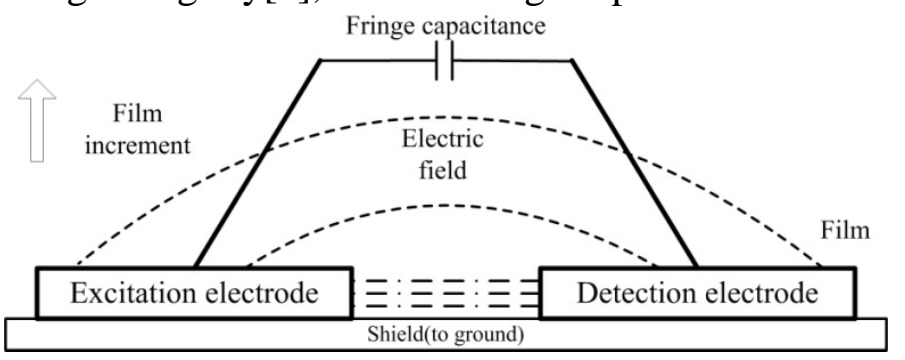

Fig. 1. Working principle of coplanar capacitive sensor

The relationship between capacitance and permittivity distribution is governed by the following equation:

$$
C=\frac{Q}{V}=-\frac{1}{V} \iint_{\Gamma} \varepsilon(x, y) \cdot[\nabla \Phi(x, y)] d \Gamma
$$

Where $\varepsilon(x, y)$ denotes the permittivity distribution in the sensing field, $V$ is the potential difference between two electrodes, $\Phi(x, y)$ corresponds to the potential distribution, $\Gamma$ refers to any surface surrounding the considered electrode.

Design parameters of coplanar capacitive sensor. The sensor performance can be evaluated from following aspects: 1) penetration depth; 2) signal strength and noise suppression; 3) measurement sensitivity and the measurement dynamic range of the sensor. The design parameters of the sensor are 1) Materials of the sensor electrodes; 2) Length of the sensor electrodes 3) Gap between the electrodes. 4) Width of the sensor electrodes.

Orthogonal experimental design. Experimental setups are shown in Fig. 2. The system consists of impedance Agilent 4294A, a computer, and coplanar capacitive sensor. AC source is set as 0.5V. Length $L$, width of electrode $W$, and gaps between the electrodes $S$ are three parameters for sensor optimization. As shown in Fig. 2c)

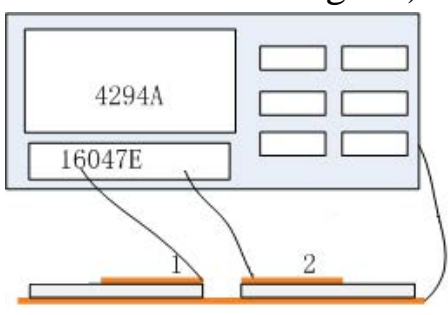

a)

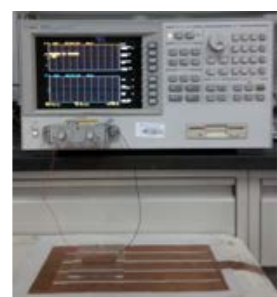

b)

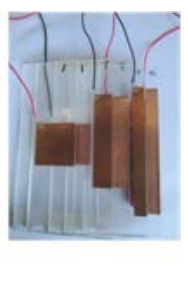

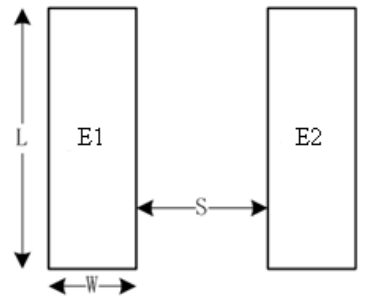

c)

Fig. 2. The block diagram and equioment of experiment

a) The block diagram b) The experimental equipments c) The top view of capacitance sensor The expression is $L_{m}\left(u^{v}\right)$, where $m$ is the number of experiments, $u$ refers to the number of parameter combination, called level, and $v$ is the number of parameters, called factors. For three-parameter experiment, the orthogonal table can be designed as shown in Table 1.

Table 1 The orthogonal table of $L_{9}\left(3^{4}\right)$ and data processing

\begin{tabular}{cccccc}
\hline & & \multicolumn{2}{c}{ Factors } & & $\begin{array}{c}\text { Value } \\
\text { derived } \\
\text { No. }\end{array}$ \\
\cline { 2 - 6 } & $\mathbf{A}$ & $\mathbf{B}$ & $\mathbf{C}$ & $\mathbf{D}$ & $\begin{array}{c}\text { by Object } \\
\text { function }\end{array}$ \\
\hline 1 & $\mathrm{~A}_{1}$ & $\mathrm{~B}_{1}$ & $\mathrm{C}_{1}$ & $\mathrm{D}_{1}$ & $y_{1}$ \\
2 & $\mathrm{~A}_{1}$ & $\mathrm{~B}_{2}$ & $\mathrm{C}_{2}$ & $\mathrm{D}_{2}$ & $y_{2}$ \\
3 & $\mathrm{~A}_{1}$ & $\mathrm{~B}_{3}$ & $\mathrm{C}_{3}$ & $\mathrm{D}_{3}$ & $y_{3}$ \\
4 & $\mathrm{~A}_{2}$ & $\mathrm{~B}_{1}$ & $\mathrm{C}_{2}$ & $\mathrm{D}_{3}$ & $y_{4}$ \\
5 & $\mathrm{~A}_{2}$ & $\mathrm{~B}_{2}$ & $\mathrm{C}_{3}$ & $\mathrm{D}_{1}$ & $y_{5}$ \\
6 & $\mathrm{~A}_{2}$ & $\mathrm{~B}_{3}$ & $\mathrm{C}_{1}$ & $\mathrm{D}_{2}$ & $y_{6}$ \\
7 & $\mathrm{~A}_{3}$ & $\mathrm{~B}_{1}$ & $\mathrm{C}_{3}$ & $\mathrm{D}_{2}$ & $y_{7}$ \\
\hline
\end{tabular}




\begin{tabular}{cccccc}
\hline 8 & $\mathrm{~A}_{3}$ & $\mathrm{~B}_{2}$ & $\mathrm{C}_{1}$ & $\mathrm{D}_{3}$ & $y_{8}$ \\
9 & $\mathrm{~A}_{3}$ & $\mathrm{~B}_{3}$ & $\mathrm{C}_{2}$ & $\mathrm{D}_{1}$ & $y_{9}$ \\
$\bar{k}_{1}$ & $\bar{k}_{11}=\left(y_{1}+y_{2}+y_{3}\right) / 3$ & $\bar{k}_{12}=\left(y_{1}+y_{4}+y_{7}\right) / 3$ & $\bar{k}_{13}=\left(y_{1}+y_{6}+y_{8}\right) / 3$ & $\bar{k}_{14}=\left(y_{1}+y_{5}+y_{9}\right) / 3$ & \\
$\bar{k}_{2}$ & $\bar{k}_{21}=\left(y_{4}+y_{5}+y_{6}\right) / 3$ & $\bar{k}_{22}=\left(y_{2}+y_{5}+y_{8}\right) / 3$ & $\bar{k}_{23}=\left(y_{2}+y_{4}+y_{9}\right) / 3$ & $\bar{k}_{24}=\left(y_{2}+y_{6}+y_{7}\right) / 3$ & \\
$\bar{k}_{3}$ & $\bar{k}_{31}=\left(y_{7}+y_{8}+y_{9}\right) / 3$ & $\bar{k}_{32}=\left(y_{3}+y_{6}+y_{9}\right) / 3$ & $\bar{k}_{33}=\left(y_{3}+y_{5}+y_{7}\right) / 3$ & $\bar{k}_{34}=\left(y_{3}+y_{4}+y_{8}\right) / 3$ & \\
Range & $R_{1}=\max \left(\bar{k}_{11}, \bar{k}_{21}, \bar{k}_{31}\right)$ & $R_{2}=\max \left(\bar{k}_{12}, \bar{k}_{22}, \bar{k}_{32}\right)$ & $R_{3}=\max \left(\bar{k}_{13}, \bar{k}_{23}, \bar{k}_{33}\right)$ & $R_{4}=\max \left(\bar{k}_{14}, \bar{k}_{24}, \bar{k}_{34}\right)$ & \\
& $-\min \left(\bar{k}_{11}, \bar{k}_{21}, \bar{k}_{31}\right)$ & $-\min \left(\bar{k}_{12}, \bar{k}_{22}, \bar{k}_{32}\right)$ & $-\min \left(\bar{k}_{13}, \bar{k}_{23}, \bar{k}_{33}\right)$ & $-\min \left(\bar{k}_{14}, \bar{k}_{24}, \bar{k}_{34}\right)$ & \\
\hline
\end{tabular}

A、B、C、D in Table 1 are four factors. In this paper, three parameters need to be considered, if three levels are considered, only 9 experiments are needed. Compared with 27 experiments are required by using full coordination method, the number of required experiment is reduced by $66 \%$. Number 1 9 in Table 1 means 9 groups of orthogonal experiment combination. The last column $\left(y_{1}-y_{9}\right)$ is value derived by object function. $\bar{k}_{1}, \bar{k}_{2}$ and $\bar{k}_{3}$ can be used to evaluate the best parameter combination according to object function. For $\bar{k}_{1}, \bar{k}_{2}$ and $\bar{k}_{3}$, each column has greatest range, expressed as $R_{i}$. It can be used to evaluate the effect weight of different factors(parameters) on sensor performance. The parameter corresponding to the largest $R_{i}$ at the same column is first order factor, the second largest $R_{i}$ determines the second order factor, and so on.

According to orthogonal experimental design principle, we set three levels, and the area of electrodes is set as $625 \mathrm{~mm}^{2}$. The ratios of length to width are $5: 1,10: 1$ and $15: 1$, and detailed setups are shown in Table 2. In order to facilitate the production, the sensor area of the three groups is slightly different, and the error is within $1.4 \%$. The level-factor table is shown in Table 3, where the factors are $A(L / W), B(S / W)$ and the frequency of excitation $f$.

Table 2. Sensor design parameters

\begin{tabular}{|c|c|c|c|}
\hline \multirow[b]{2}{*}{ Groups } & \multicolumn{3}{|c|}{ Parameters } \\
\hline & $\begin{array}{c}\text { Length of electrode } \\
\qquad(\mathrm{mm})\end{array}$ & $\begin{array}{c}\text { Width of electrode } \\
\text { W(mm) }\end{array}$ & $\begin{array}{c}\text { Sensor area } \\
\mathrm{Sa}\left(\mathrm{mm}^{2}\right)\end{array}$ \\
\hline 1 & 56.0 & 11.2 & $625+2.20$ \\
\hline 2 & 79.0 & 7.9 & $625-0.90$ \\
\hline 3 & 97.5 & 6.5 & $625+8.75$ \\
\hline
\end{tabular}

Table 3. The parameters of factors and level

\section{Factors}

\begin{tabular}{cccc}
\cline { 2 - 4 } Levels & $\begin{array}{c}\text { Ratio of length to width } \\
\boldsymbol{A}(\boldsymbol{L} / \boldsymbol{W})\end{array}$ & $\begin{array}{c}\text { Ratio of gaps to width } \\
\boldsymbol{B}(\boldsymbol{S} / \boldsymbol{W})\end{array}$ & $\begin{array}{c}\text { Frequency of } \\
\text { excitation } \\
\boldsymbol{f}(\mathbf{M H z})\end{array}$ \\
\hline 1 & $A_{1}(5)$ & $B_{1}(1)$ & $f_{1}(0.5)$ \\
2 & $A_{2}(10)$ & $B_{2}(2)$ & $f_{2}(1.0)$ \\
3 & $A_{3}(15)$ & $B_{3}(3)$ & $f_{3}(1.5)$ \\
\hline
\end{tabular}

Orthogonal design table are using $L_{9}\left(3^{4}\right)$ table, as shown in Table 4. The evaluation standards are measured capacitance and simulation results, respectively. The experiment and simulation results in the double vertical coordinate system are given in Fig. 3. The left side of the vertical coordinate represents the experimental result, and the right side of the vertical coordinate is the simulation result.

Table 4 Experimental scheme and results with the same area electrodes

\begin{tabular}{cccccc}
\hline \multirow{2}{*}{ Experiments } & \multicolumn{3}{c}{ Factors } & \multicolumn{2}{c}{ Capacitance (pF) } \\
\cline { 2 - 6 } & \multirow{2}{*}{$\boldsymbol{A}(\boldsymbol{L} / \boldsymbol{W})$} & $\boldsymbol{B}(\boldsymbol{S} / \boldsymbol{W})$ & $\boldsymbol{f}(\mathbf{M H z})$ & $\begin{array}{c}\text { Measurements } \\
\boldsymbol{y}_{\mathbf{m}}\end{array}$ & $\begin{array}{c}\text { Simulations } \\
\boldsymbol{y}_{\mathbf{s}}\end{array}$ \\
\hline 1 & $A_{1}$ & $B_{1}$ & $f_{1}$ & 0.189 & 44.64 \\
2 & $A_{1}$ & $B_{2}$ & $f_{2}$ & 0.138 & 23.36 \\
3 & $A_{1}$ & $B_{3}$ & $f_{3}$ & 0.173 & 15.82 \\
4 & $A_{2}$ & $B_{1}$ & $f_{2}$ & 0.291 & 84.32 \\
5 & $A_{2}$ & $B_{2}$ & $f_{3}$ & 0.150 & 44.72 \\
\hline
\end{tabular}




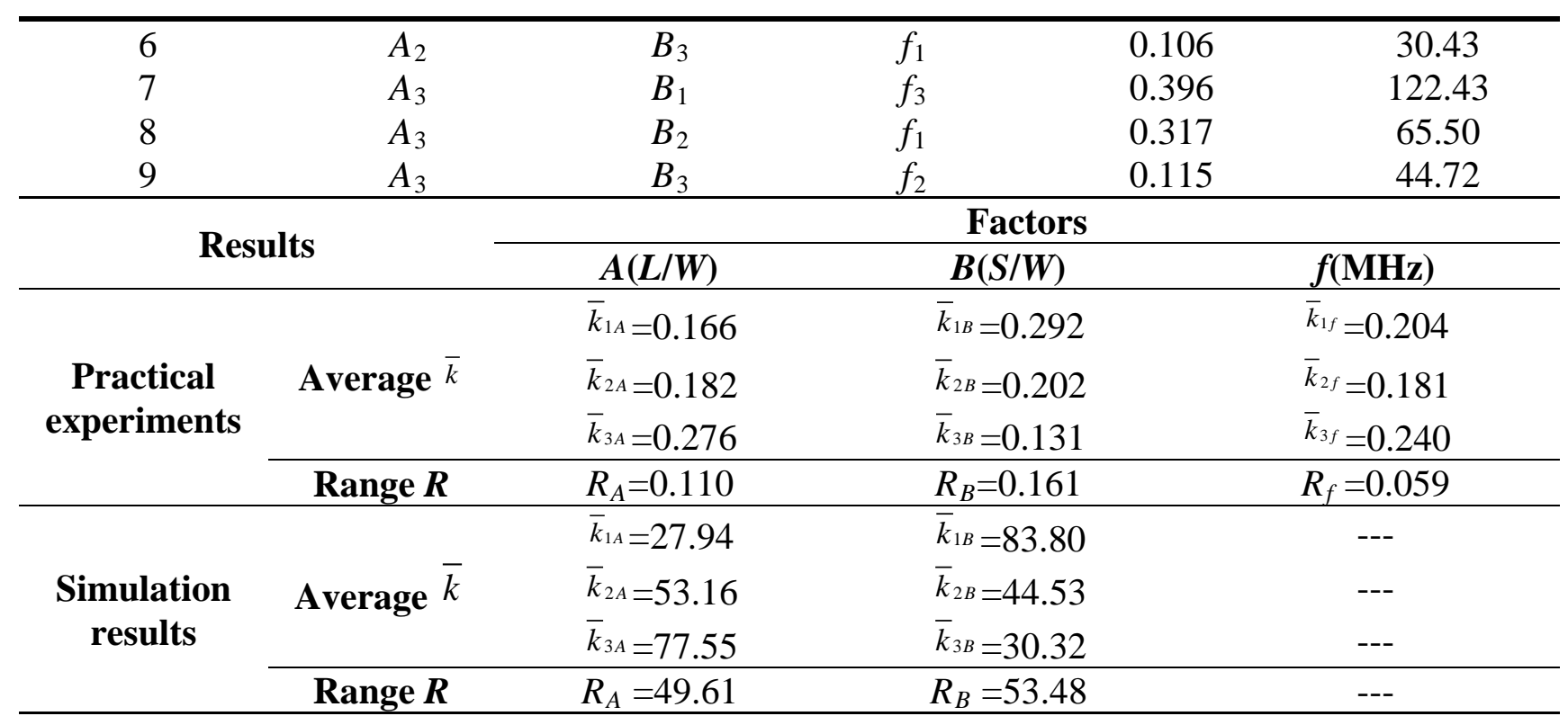

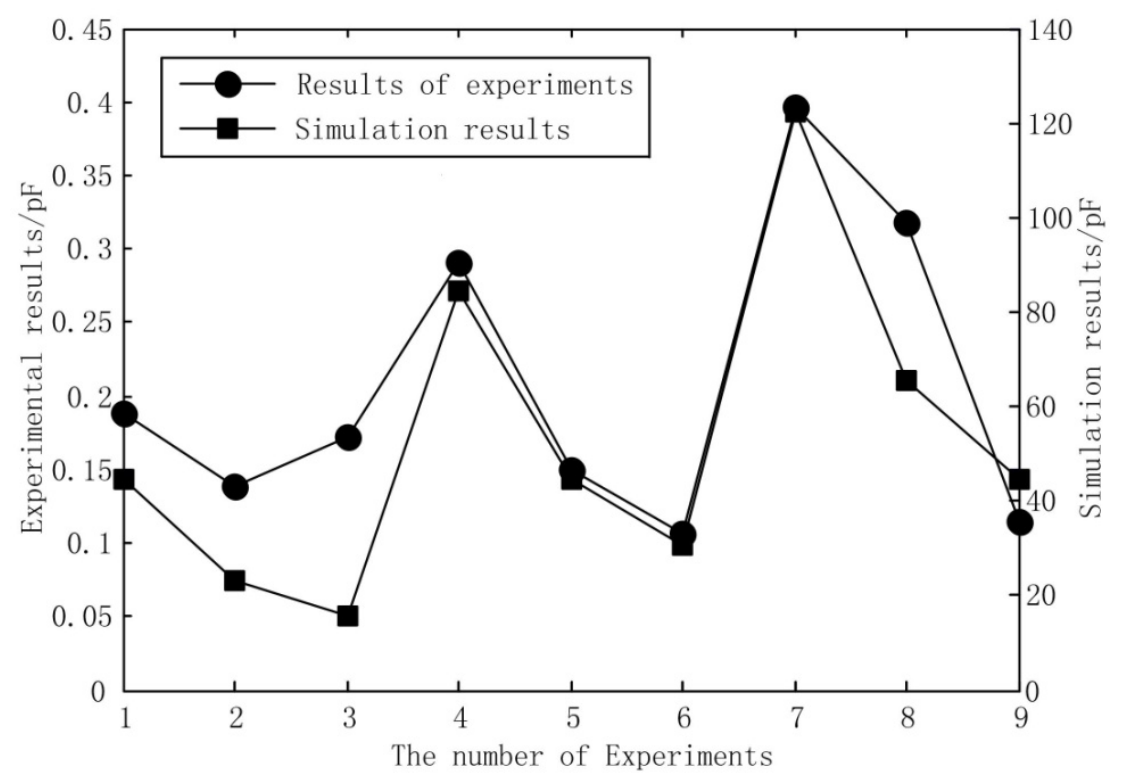

Fig. 3. Experiment and simulation results

From Fig. 3, we can derive that the results of experiment and simulation are of great difference, and the difference is almost three orders of magnitude, however, the curve trends are similar. The reasons of this situation are mainly from two aspects, 1) Excitation frequency cannot be set in the case of simulation, because the simulation mode of COMSOL is electrostatic mode. However, we set excitation frequencies $500 \mathrm{kHz}, 1 \mathrm{MHz}$ and $1.5 \mathrm{MHz}$, respectively when we process the practical experiments. 2) circumstance conditions may affect the final measurement results which lead to reduced measurement results. From Table 4, we note $R_{B}>R_{A}$, which means the influence of factor $S / W$ on sensor signal strength is greater than factor $S / W . R_{f}$ is the smallest range, which means the effect of excitation frequency on sensor performance is minimum.

Table 5 indicates the best and the worst combination of sensor design parameters. To prove its effectiveness, supplementary experiments are carried out, the measured capacitance value is $0.098 \mathrm{pF}$, which is the smallest capacitance. Therefore, the worst combination is $A_{3} B_{1} f_{3}$. According to orthogonal design of simulation results, the best and the worst combination are $A_{3} B_{1}$ and $A_{1} B_{3}$, respectively. This result is same as the result shown in practical experiments. 
Table 5. Experimental result processing with the same area electrodes

\begin{tabular}{ccccc}
\hline \multirow{2}{*}{ Combinations } & \multicolumn{2}{c}{ Practical experiments(pF) } & \multicolumn{2}{c}{ Simulation results(pF) } \\
\cline { 2 - 5 } & $\begin{array}{c}\text { 9 groups of } \\
\text { experiments }\end{array}$ & Final results & $\begin{array}{c}\text { 9 groups of } \\
\text { experiments }\end{array}$ & $\begin{array}{c}\text { Final } \\
\text { results }\end{array}$ \\
\hline $\begin{array}{c}\text { The best } \\
\text { combination } \\
\text { The worst } \\
\text { combination }\end{array}$ & $A_{3} B_{1} f_{3}$ & $A_{3} B_{1} f_{3}$ & $A_{3} B_{1}$ & $A_{3} B_{1}$ \\
\hline
\end{tabular}

\section{Conclusions}

The main conclusion is listed as follows:

(1)Orthogonal experimental design can improve the efficient of experiments, and the number of experiments can be reduced around $66 \%$.

(2) The optimal combinations of sensor design parameters are derived, which indicate the primary parameter is gaps of sensor electrode, the secondary parameter is length of sensor electrode, and the last is electrode width.

\section{Acknowledgements}

This work is financially supported by the Project 51475013 and 51105008 National Natural Science Foundation of China.

\section{References}

[1] Kuirong Xiong, Deru Ni. Sensor World, No.03, pp.16-19, 1998. (In Chinese)

[2] Nan Li, Baolong Guo, Pai Wang. Journal of Mechanical Engineering, Vol.46, No.14, pp12-17, 2010. (In Chinese)

[3] K.Sundara-Rajan, L.Byrd, A.V.Mamishev. IEEE Sensors Journal, Vol.4, No.3, pp.378-383, 2004.

[4] Yu Pang, Ensheng Dong, Yunjing Wu, et al. Metrology \& Measurement Technology, Vol.30, No.6, pp.11-13, 2010. (In Chinese)

[5] Zheng Liu, Shaogang Liu, Yuesheng Luo, et al. IEEE International Conference on Information and Automation, Harbin, Heilongjiang. pp.912-916, 2010.

[6] Xiaoming Chang, Yinke Dou, Dun Zhou, et al. International Conference on Computing, Measurement, Control and Sensor Network, Taiyuan, Shanxi. pp.417-420, 2012.

[7] Yu Gong, Hai Ye Zhu, Nan Li., Advanced Materials Research, 945-949, pp2030-2036, 2014. 